\title{
Linking structural and electronic properties of high-purity self-assembled GaSb/GaAs quantum dots
}

\author{
T. Nowozin, ${ }^{*}$ A. Marent, L. Bonato, A. Schliwa, and D. Bimberg ${ }^{\dagger}$ \\ Institut für Festkörperphysik, Technische Universität Berlin, Hardenbergstrasse 36, 10623 Berlin, Germany \\ E. P. Smakman, J. K. Garleff, and P. M. Koenraad \\ Department of Applied Physics, Eindhoven University of Technology, P.O. Box 513, 5600 MB Eindhoven, The Netherlands \\ R. J. Young and M. Hayne \\ Department of Physics, Lancaster University, Lancaster LAI 4YB, United Kingdom \\ (Received 19 March 2012; revised manuscript received 18 June 2012; published 6 July 2012)
}

\begin{abstract}
We present structural, electrical, and theoretical investigations of self-assembled type-II GaSb/GaAs quantum dots (QDs) grown by molecular beam epitaxy. Using cross-sectional scanning tunneling microscopy (X-STM) the morphology of the QDs is determined. The QDs are of high purity ( $\sim 100 \% \mathrm{GaSb}$ content) and have most likely the shape of a truncated pyramid. The average heights of the QDs are 4-6 $\mathrm{nm}$ with average base lengths between 9 and $14 \mathrm{~nm}$. Samples with a QD layer embedded into a $p n$-diode structure are studied with deep-level transient spectroscopy (DLTS), yielding a hole localization energy in the QDs of $609 \mathrm{meV}$. Based on the X-STM results the electronic structure of the QDs is calculated using 8-band $\mathbf{k} \cdot \mathbf{p}$ theory. The theoretical localization energies are found to be in good agreement with the DLTS results. Our results also allow us to estimate how variations in size and shape of the dots influence the hole localization energy.
\end{abstract}

DOI: 10.1103/PhysRevB.86.035305

PACS number(s): 73.21.La, 68.65.Hb, 85.35.Be

\section{INTRODUCTION}

$\mathrm{GaSb} / \mathrm{GaAs}$ quantum $\operatorname{dots}^{1}(\mathrm{QDs})$ are very particular due to their type-II band alignment. The spatial separation of electrons and holes in type-II structures results in long exciton lifetimes, ${ }^{2-4}$ resulting in interesting applications for longwavelength optoelectronics. ${ }^{5}$ The exclusive confinement of holes and their large localization energy makes $\mathrm{GaSb} / \mathrm{GaAs}$ QDs particularly interesting for charge storage devices. ${ }^{6-8}$

$\mathrm{GaSb} / \mathrm{GaAs}$ QDs were first grown using molecular beam epitaxy ${ }^{2,9}$ and later by metal-organic chemical vapor epitaxy, ${ }^{10,11}$ followed by optical characterization, ${ }^{9,12-15}$ cross-section scanning electron microscopy investigations, ${ }^{16}$ and deep-level transient spectroscopy (DLTS) studies, ${ }^{17,18}$ accompanied by numerical calculations. ${ }^{19-21}$ The various investigations have been performed on different samples. A clear link between the structural properties and the hole localization energy as the key electronic property has not been established so far.

Linking structural and electronic properties of selforganized quantum dots involves four major challenges:

(1) The first one is connected to the intricate problem of measuring the morphology of the QDs in 3D. Although there is presently no method available that discloses completely the atom-by-atom ordering of the complete structure in 3D, cross-sectional scanning tunneling microscopy is presently the best approximation solving this problem. It delivers all eminent structural information of the QD with atomistic resolution that is available at the QD's cleavage plane.

(2) The second one is related to the experimental determination of the electronic structure. For type-I quantum dots, such as InAs/GaAs, excitonic and multiexcitonic properties, measured by high-resolution photoluminescence, yield a wealth of information about confined electrons and holes and their Coulomb interaction. ${ }^{22}$ For type-II quantum dots, where electrons and holes are spatially separated, the interpretation of PL spectra, in particular the identification of the various peaks, is intrinsically difficult. The key information for memory applications, namely the hole-activation energy, cannot be extracted reliably. Therefore, the methods of choice, as applied in this work, are electrical methods, such as capacitancevoltage $(\mathrm{C}-\mathrm{V})$ and DLTS measurements.

(3) The third challenge is to correlate structural and spectroscopic investigations. The QD ensemble exhibits a certain variation in the morphological properties of the individual QDs. The most obvious approach-combining structural and electrical characterization for one and the same QD-is not feasible at present. However, it is already a big step forward to link results from samples which have the same growth parameters for the QD layer, as is done here.

(4) The fourth task involves electronic structure calculations. In general, such calculations start out from a 3D model quantum dot structure including the distribution of various atomic species, continue with the incorporation of strain and piezoelectricity, yielding the energy levels and the optical spectrum. Obviously, electronic structure calculation links structural and electronic properties. This link is of tremendous value for device design, provided that the relationship is based on a reliable model for the electronic structure calculations. In this work we employ our previously successful 3D implementation of the 8-band $\mathbf{k} \cdot \mathbf{p}$ envelope function theory ${ }^{23,24}$ which includes strain and piezoelectricity. The predictive power of this method, however, critically depends on the quality of the material parameters entering the simulations, here in particular, the band offsets between GaAs and GaSb. Our results thus contribute to discrimination between a variety of material parameters and calibrate the electronic structure simulations. 
We pursue the following strategy to establish the link between structural and electronic properties. Two samples are grown by MBE using identical growth conditions for the QD layer. The first sample contains multiple layers of QDs and is used for X-STM measurements. In the second sample a single QD layer is inserted in a $p n$-diode to perform electrical measurements. Using DLTS, the mean activation energy and the capture cross section, and by employing charge-selective DLTS, the hole localization energy, are determined as key electronic properties of the QDs. Next, the information obtained from the structural investigation is used as input for the 8-band $\mathbf{k} \cdot \mathbf{p}$-calculations. Finally, the localization energies measured by DLTS are compared to the results of the calculations. A third sample contains only a wetting layer inside the $p n$-diode, and is used as general reference for the DLTS measurements.

\section{SAMPLES}

The GaSb quantum dots are grown in an upgraded $\mathrm{VH}$ V80H MBE reactor. The growth conditions for the dots, such as the cold cap thickness or the maximum substrate temperature after the dot formation, were systematically altered. ${ }^{25}$ From that series, the parameters yielding "best" QD formation were selected for the samples investigated here. The growth procedure was the following: First, a GaAs buffer was deposited at a pyrometer-calibrated temperature of $600{ }^{\circ} \mathrm{C}$, then a growth interrupt followed for 1 minute after a 3-minute cool-down to $480{ }^{\circ} \mathrm{C}$ under $\mathrm{As}_{2}$. Then the $\mathrm{Sb}$ shutter was opened and after $2 \mathrm{~s} \mathrm{GaSb}$ deposition of nominally $2.1 \mathrm{ML}$ was started for $7 \mathrm{~s}$ at a temperature of $480{ }^{\circ} \mathrm{C}$. Next, the substrate was cooled down to $370{ }^{\circ} \mathrm{C}$ under $\mathrm{Sb}$ flux for 4 minutes. The As shutter was then opened and after 2 s a $20 \mathrm{~nm}$ GaAs cold cap was deposited at the same temperature. Finally, GaAs was grown on top of the cold cap with the temperature not exceeding $500{ }^{\circ} \mathrm{C}$.

The X-STM sample contains multiple layers of these QDs. For the DLTS measurements two samples were grown consisting of a $n^{+} p$-diode structure with one embedded layer of $\mathrm{GaSb}$ (see Fig. 1). In one of the two samples, which will further be referred to as the $Q D$ sample, nominally $2.1 \mathrm{ML}$ of $\mathrm{GaSb}$ were deposited until GaSb QDs with a nominal density of $\sim 5 \times 10^{10} \mathrm{~cm}^{-2}$ formed. In the other sample, which will further be referred to as the WL sample, only 0.9 ML of GaSb were deposited such that only the wetting layer formed.

The $p n$-diode structures incorporate the following sequence: On top of a semi-insulating GaAs substrate, a 150-nmthick GaAs buffer layer, a 500-nm-thick highly $p$-doped (Be, $\left.p=2 \times 10^{18} \mathrm{~cm}^{-3}\right) \mathrm{GaAs}$ contact layer, and a 700-nm-thick $p$-doped $\left(\mathrm{Be}, p=2 \times 10^{16} \mathrm{~cm}^{-3}\right.$ ) GaAs layer were deposited. Then, a 20 -nm-thick $\mathrm{Al}_{0.1} \mathrm{Ga}_{0.9}$ As layer was grown to prevent the diffusion of Be into the layers above (which would have adverse effects on the QD quality). Subsequently, the GaSb layer was embedded into 7-nm nominally undoped GaAs on either side, followed by another $500 \mathrm{~nm}$ of $p$-doped $(\mathrm{Be}, p=$ $2 \times 10^{16} \mathrm{~cm}^{-3}$ ) GaAs. Finally, a 400-nm-thick highly $n$-doped (Te, $n=1 \times 10^{18} \mathrm{~cm}^{-3}$ ) contact layer was deposited. Using standard optical lithography techniques $400-\mu \mathrm{m}$-wide round mesa structures were defined and Ohmic $n$-type $(\mathrm{Ni} / \mathrm{AuGe} / \mathrm{Au})$ and $p$-type $(\mathrm{Ti} / \mathrm{Pt} / \mathrm{Au})$ contacts are formed on the highly

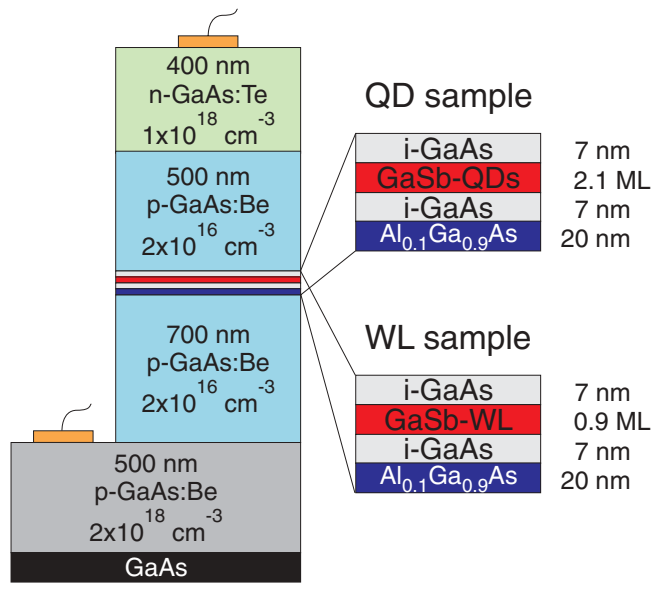

FIG. 1. (Color online) Sample structure for the DLTS measurements. The QD sample contains one layer of $\mathrm{GaSb} / \mathrm{GaAs}$ QDs embedded into the $p$-doped region of a $p n$-diode while the WL sample contains only the WL as GaSb deposition is stopped before QD formation.

doped contact layers by thermal evaporation of the metals and subsequent alloying.

\section{STRUCTURAL INVESTIGATIONS}

The morphology of the QDs was investigated using X-STM (details on the measurements can be found in Smakman et $\left.a l .^{25}\right)$. For the growth conditions described in Sec. II, mainly truncated pyramidal QDs [VAR $<0.5$, vertical aspect ratio VAR defined as height divided by base length, see Fig. 2(e)] are observed. Qualitative assessment shows that these are high-purity GaSb dots, which show almost no intermixing with the surrounding GaAs lattice; e.g., see Fig. 2(a). Because of surface relaxation, the locally highly strained GaSb QDs appear higher and therefore brighter in the X-STM measurements, which allows the determination of their shape and composition. Therefore, a detailed study of the QD height and width is possible with this technique. A selection of three QDs out of a set of 42 analyzed is shown in Fig. 2(a). The apparent shape of the QDs in the sample is pyramidal with the majority being truncated-pyramidal. A density plot of the apparent height versus the apparent base length of the QDs measured is displayed in Fig. 2(b). As the lateral position at which the sample is cleaved ( $\{110\}$ plane) is random, the measured values overestimate the VAR and the scattering of the sizes (see Sec. V). ${ }^{26}$ Hence, the real vertical aspect ratio is expected to be lower than the apparent one. Figures 2(c) and 2(d) show histograms of the measured base lengths and heights, respectively. The base lengths cluster around $11 \mathrm{~nm}$ with the majority of QDs lying within a margin of $\pm 6 \mathrm{~nm}$ of this value. Above a base length of about $18 \mathrm{~nm}$ QDs are sporadically found. We attribute the former group to strained QDs and the latter to relaxed QDs. The majority of QDs measured have a height of about 4-6 nm. Qualitative measurements indicate a very high $\mathrm{GaSb}$ content within the QDs of $\sim 100 \%$. 

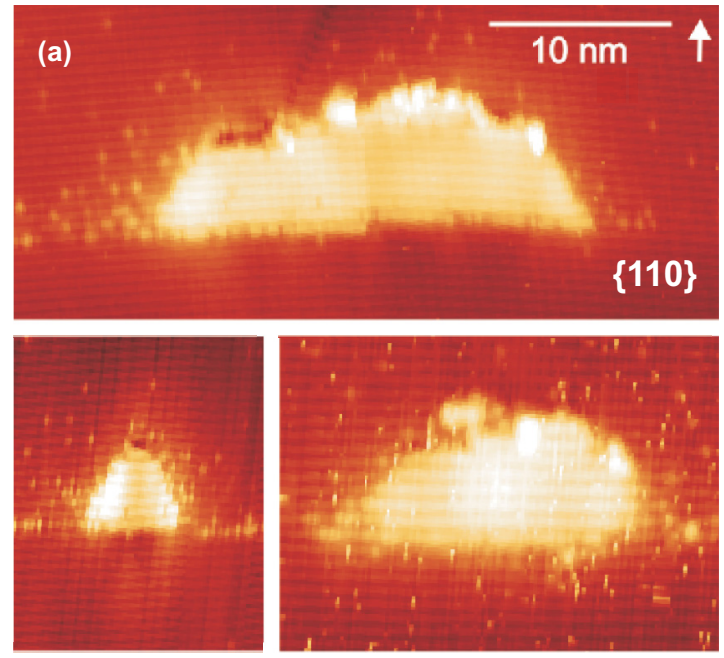

Base Length $(\mathrm{nm})$
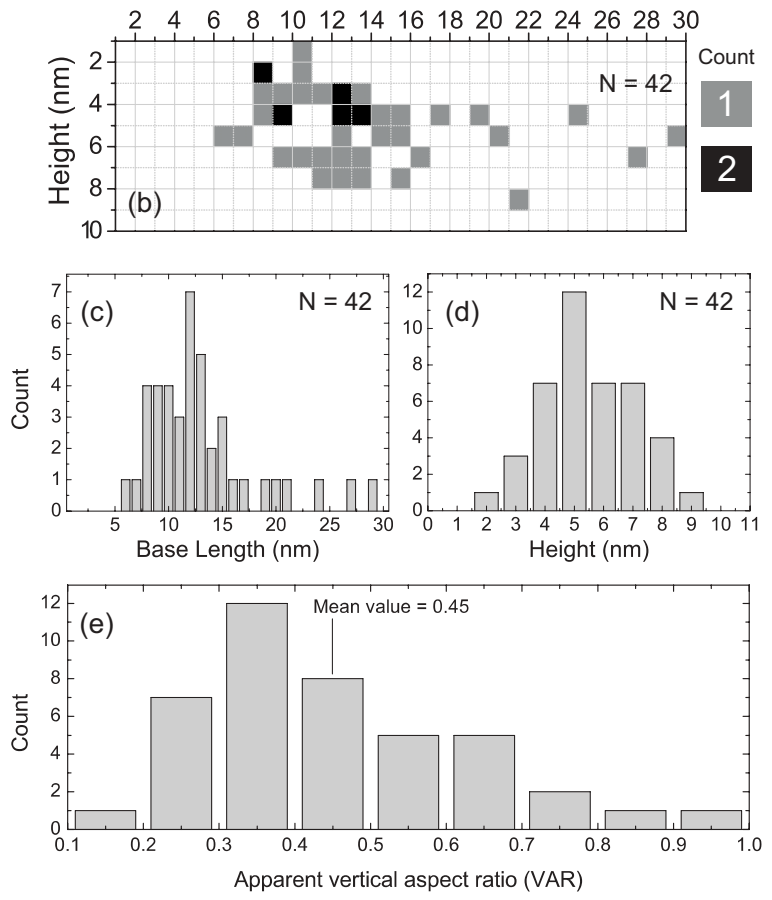

FIG. 2. (Color online) Cross-sectional scanning tunneling microscope (X-STM) measurements on a total of $N=42$ quantum dots cleaved at a $\{110\}$ plane. (a) GaSb QDs have a pyramidal or truncated pyramidal shape. (b) Base length and height distribution. (c) Base length histogram (d) Height histogram. (e) Apparent vertical aspect ratios determined from the values in (b).

\section{ELECTRICAL INVESTIGATION}

For the application of GaSb/GaAs QDs in novel memories the hole energy levels are of crucial importance. C-V measurements and in particular DLTS measurements ${ }^{27-29}$ are the best methods to obtain detailed information about their position. Previously, there have been only two DLTS studies of the GaSb/GaAs QD system to the best of our knowledge. One $^{17}$ suffered from poor sample quality, while the other ${ }^{18}$ estimated a hole localization energy of $450 \mathrm{meV}$ in QDs grown by MOCVD, which means under completely different conditions. During MOCVD an additional chemical reaction

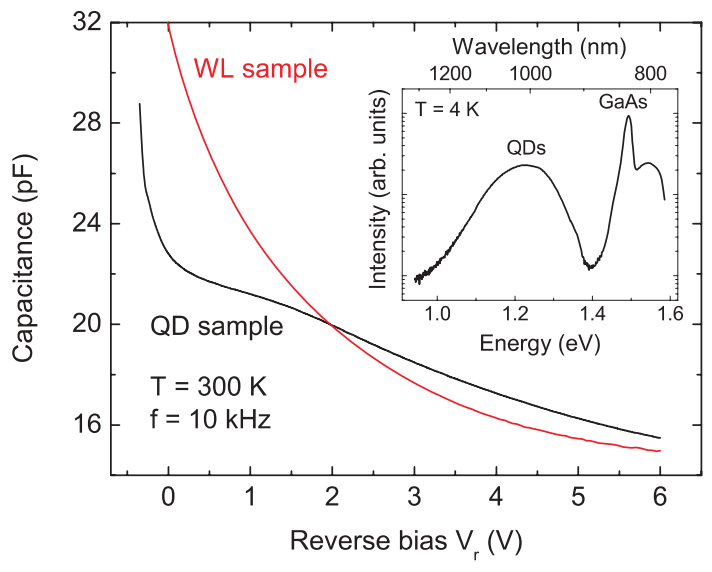

FIG. 3. (Color online) C-V curves of the QD and the WL samples at $300 \mathrm{~K}$ and $f=10 \mathrm{kHz}$. The QD sample shows a plateau-like feature due to the additional capacitance of the QD ensemble. The inset shows the PL graph of the QD sample at $4 \mathrm{~K}$ and an excitation power of $10 \mathrm{~mW}$ on an area of about $1 \mathrm{~mm}^{2}$ at $532 \mathrm{~nm} \mathrm{CW}$.

has to take place before the surface reaction. Sb tends to form pure crystals, and in order to prevent that, the partial pressure has to be kept low, which is much more easily achieved in MBE than in MOCVD. Hence, the QDs grown by MOCVD can be expected to be less pure than the ones grown by MBE. A lower localization energy would be the result.

\section{A. C-V measurements}

Figure 3 compares the $\mathrm{C}-\mathrm{V}$ measurements of the QD and the WL samples. Due to the additional capacitance of the QD ensemble a plateau-like feature appears between a reverse bias of $V_{r}=-0.3$ and $V_{r}=2 \mathrm{~V}$ in the C-V characteristics of the QD sample. The WL sample does not show this feature. The beginning and the end of the plateau indicate the reverse bias voltages at which the QD ensemble contributes to the capacitance. The inset of Fig. 3 shows the PL of the QD sample at a temperature of $4 \mathrm{~K}$ and an excitation power of $10 \mathrm{~mW}$ of an area of about $1 \mathrm{~mm}^{2}$ at $532 \mathrm{~nm} \mathrm{CW}$ excitation. A broad peak due to the recombination in the QD ensemble appears around $1.22 \mathrm{eV}$ with a FWHM of $\sim 140 \mathrm{meV}$.

\section{B. Conventional DLTS}

Next, we measure the activation energy of holes in QDs. For this purpose, we employ a method that was originally developed for the measurement of activation energies of deep levels. ${ }^{27}$ In such a standard DLTS experiment the QDs are filled with holes for $500 \mathrm{~ms}$ at a voltage of $V_{\text {pulse }}=0 \mathrm{~V}$. Then, the emission transient is recorded at a reverse bias of $V_{r}=3 \mathrm{~V}$. Under these conditions the QDs are completely filled during the pulse and emit all the holes at the measurement voltage. Due to ensemble broadening and many-particle effects the emission transients are not monoexponential. Hence the transients are analyzed with the double-boxcar method. ${ }^{30,31}$ The work cycle is repeated for different temperatures and results in a temperature-dependent curve, which shows maxima at those temperatures where the emission time constant coincides with the reference time constant $\tau_{\text {ref }}$. The DLTS spectra for the QD sample and the WL sample are shown in Fig. 4 for a reference 


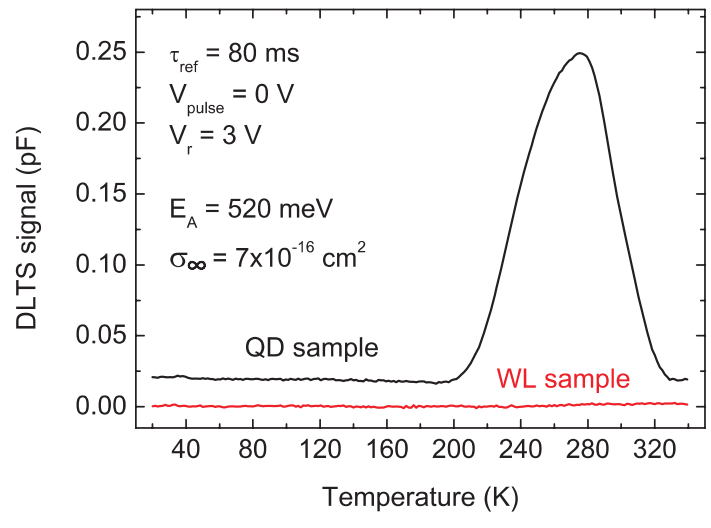

FIG. 4. (Color online) DLTS spectrum of the QD and the WL sample for a reference time constant of $\tau_{\text {ref }}=80 \mathrm{~ms}$. The QD sample shows a clear DLTS signal around a temperature of $270 \mathrm{~K}$ while the WL sample shows no signal throughout the whole temperature range. The data of the QD sample is offset by $20 \mathrm{fF}$ for clarity.

time constant of $\tau_{\text {ref }}=80 \mathrm{~ms}$. A clear peak can be seen for the QD sample around a temperature of $270 \mathrm{~K}$ while the WL sample shows no signal throughout the whole temperature range.

As tunneling processes are negligible in this voltage range, the holes trapped in the QDs are emitted only due to thermal excitation. In this case, the thermal emission rate $e_{a}$ is ${ }^{27}$

$$
e_{a}=\gamma T^{2} \sigma_{\infty} \exp \left(-E_{a} / k T\right),
$$

where $E_{a}$ is the activation energy, $T$ the temperature, $k$ the Boltzmann constant, $\sigma_{\infty}$ the capture cross section for $T=\infty$, and $\gamma$ a temperature-independent constant. From an Arrhenius plot of the DLTS peak of the QD sample at different reference time constants we obtain an activation energy of $E_{a}=520 \mathrm{meV}$ and a capture cross section of $\sigma_{\infty}=7 \times 10^{-16} \mathrm{~cm}^{2}$. The peak in Fig. 4 is the result of the complete discharging of initially full QDs during the measurement, where the activation energy of each hole level depends on the charge state of the QD. Hence the DLTS peak is broadened. ${ }^{18,32}$ The activation energy derived from the Arrhenius plot represents the mean activation energy for all hole levels of the QD ensemble including the additional $\mathrm{Al}_{0.1} \mathrm{Ga}_{0.9} \mathrm{As}$ barrier. If we assume a value of $\Delta E_{V B}=54 \mathrm{meV}$ for the valence band offset (based on a 66:34 split of the band gap difference ${ }^{33}$ ) between $\mathrm{GaAs}$ and $\mathrm{Al}_{0.1} \mathrm{Ga}_{0.9} \mathrm{As}$ and subtract this value from the data, we get a mean activation energy of $\sim 466 \mathrm{meV}$ for the QD ensemble.

\section{Charge-selective DLTS}

The conventional DLTS yields the mean activation energy of all holes emitted from the QD ensemble. However, to establish the link to theory, the hole ground state activation energy (localization energy) is the important one. To study the electronic structure of the QDs in more detail we hence employ charge-selective DLTS, ${ }^{6,18}$ where the pulse voltages $V_{\text {pulse }}$ and the measurement voltages $V_{r}$ are set such that, on average, approximately only one hole per QD gets captured or emitted during the pulse. This allows us to successively probe deeper lying hole states down to the ground state. The resulting DLTS spectra for a reference time constant of $\tau_{\text {ref }}=80 \mathrm{~ms}$ are shown in Fig. 5(a) for temperatures ranging from $100 \mathrm{~K}$ to
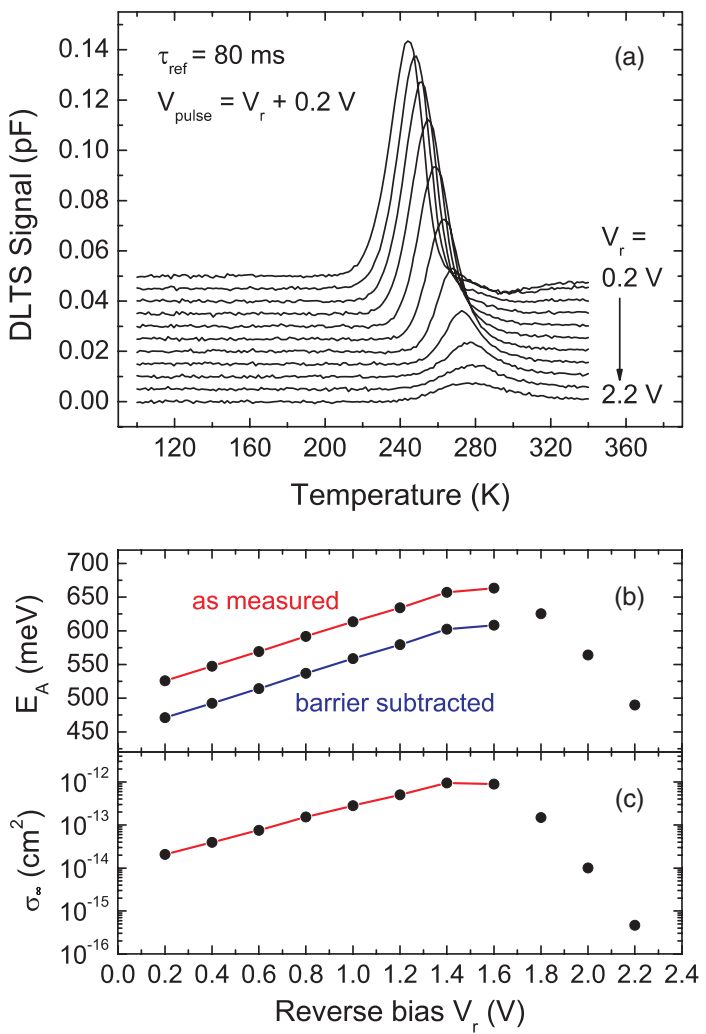

FIG. 5. (Color online) (a) Charge-selective DLTS spectra of the QD sample for a reference time constant of $\tau_{\text {ref }}=80 \mathrm{~ms}$. When the reverse bias is increased, the emission of holes from lower levels in the QDs is probed. The curves are offset by $5 \mathrm{fF}$ each for clarity. (b) Activation energies $E_{a}$ (upper red curve) and (c) apparent capture cross sections $\sigma_{\infty}$ derived from an Arrhenius plot for each reverse bias $V_{r}$. The additional activation energy of the $\mathrm{Al}_{0.1} \mathrm{Ga}_{0.9} \mathrm{As}$ barrier is subtracted (lower blue curve). The unconnected data points are not attributed to the emission of holes from QDs.

$340 \mathrm{~K}$. The pulse bias is set to $V_{\text {pulse }}=V_{r}+0.2 \mathrm{~V}$ while the measurement voltage is increased from $0.2 \mathrm{~V}$ to $2.2 \mathrm{~V}$. Due to the smaller range which is probed during the voltage pulses, the DLTS peaks are narrower than in conventional DLTS. The peaks move from about $245 \mathrm{~K}$ for $V_{r}=0.2 \mathrm{~V}$ to about $275 \mathrm{~K}$ for $V_{r}=2.2 \mathrm{~V}$.

From the shift of the DLTS peaks for different reference time constants $\tau_{\text {ref }}$ again the Arrhenius plots for the different reverse biases are obtained (not shown). From the Arrhenius plots the activation energies $E_{a}$ and the apparent capture cross sections $\sigma_{\infty}$ are obtained for each individual reverse bias $V_{r}$. The values are displayed in Figs. 5(b) and 5(c), respectively. With increasing reverse bias deeper hole levels in the QDs are probed during the measurement. Hence, the measured activation energies increase from $525 \mathrm{meV}$ for $V_{r}=0.2 \mathrm{~V}$ to $663 \mathrm{meV}$ at $V_{r}=1.6 \mathrm{~V}$, while the apparent capture cross section increases from $\sigma_{\infty}=2 \times 10^{-14} \mathrm{~cm}^{2}$ for $V_{r}=0.2 \mathrm{~V}$ to $\sigma_{\infty}=1 \times 10^{-12} \mathrm{~cm}^{2}$ at $V_{r}=1.6 \mathrm{~V}$. Normally, for such doping concentrations in the diode structure one would expect a lower activation energy for measurement voltages close to $0 \mathrm{~V}$. A reason for this deviation could be a doping concentration that is lower than the nominal one, leading to a larger depletion region on the $p$-side reaching the QDs already at a bias of $0 \mathrm{~V}$. Then, 
it is not possible to measure the emission of the highest hole states of the QD ensemble. For measurement voltages higher than $V_{r}=1.6 \mathrm{~V}$ the activation energy and the apparent capture cross sections decrease again. We attribute these effects to emission processes from defect states related to the growth of QDs (i.e., GaSb clusters, relaxed QDs) but not to the emission of holes from the QDs themselves. This is underpinned by the range in which the apparent capture cross section for the ground state of QDs is typically found $\left(\sigma_{\infty}=10^{-13} \mathrm{~cm}^{2}\right.$ to $10^{-12} \mathrm{~cm}^{2}$ ). To obtain the localization energy of the QDs we again subtract the contribution of the $\mathrm{Al}_{0.1} \mathrm{Ga}_{0.9}$ As barrier $(\Delta E=54 \mathrm{meV}){ }^{33}$ The results are represented by the blue curve in Fig. 5(b). From this curve, a localization energy of $E_{\mathrm{loc}}=609 \mathrm{meV}$ is derived for the present GaSb QDs.

\section{THEORETICAL RESULTS}

Since the GaSb/GaAs share a common cation (Ga), the hypothetical conduction-band offset in the absence of strain is very small. Hence, the band-gap difference between GaAs and $\mathrm{GaSb}$ translates almost completely to a large valence-band offset. In the presence of strain, due to the lattice mismatch, the local band structure of a real heterostructure is strongly modified, leading to a huge increase $(>800 \mathrm{meV}$ for $100 \%$ $\mathrm{GaSb}$ content ${ }^{21}$ ) of the GaSb conduction-band and a clear type-II band alignment. The dots are hence repulsive for electrons and attractive for holes.

To shed light into the relation between morphology and DLTS results, we carried out electronic structure modeling, varying size, shape, and composition of the quantum dots. Guided by our X-STM results, we took into account the real morphology and the resulting strain, including the piezoelectric fields, by using a 3D implementation of eight-band $\mathbf{k} \cdot \mathbf{p}$ envelope function theory, as outlined in Ref. 24.

Figure 6 shows the hole localization energies as function of size [Fig. 6(a)] and vertical aspect ratio [Fig. 6(b)] for $100 \%$ and $50 \% \mathrm{GaSb}$ content.

Size variation. Guided by the X-STM measurements the QD height is varied between 1.4 and $6.3 \mathrm{~nm}$, while keeping the QD shape fixed with an aspect ratio of 0.2 (due to the overestimation of the vertical aspect ratio, ${ }^{26}$ a smaller value than the mean value of the apparent vertical aspect ratio has to be used). For this large size-change the hole localization

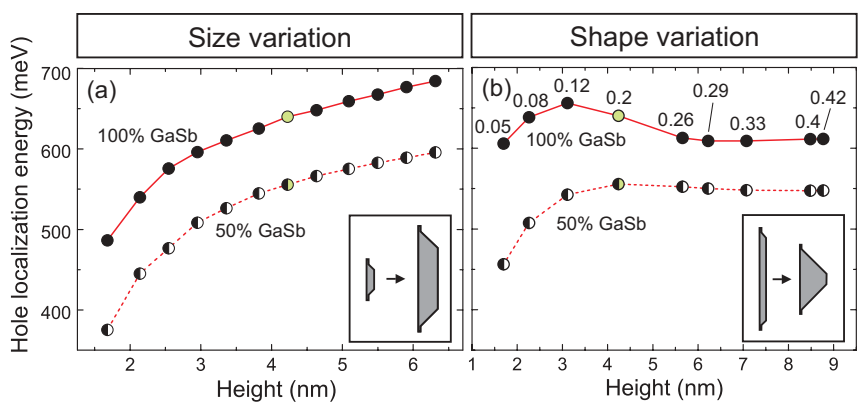

FIG. 6. (Color online) Hole localization energies as function of (a) QD size and (b) shape (expressed in terms of QD height with constant volume) and composition. The numbers indicate the VAR for the data points. The green data points are the initial start points with equal parameters for both calculation series. energies of pure GaSb QDs change from about $490 \mathrm{meV}$ for small to $685 \mathrm{meV}$ for large quantum dots. The values for $50 \%$ $\mathrm{GaSb}$ content are offset by approximately $100 \mathrm{meV}$ to lower energies. The results can be understood in terms of the quantum size effects for QDs and the smaller valence band offset for decreasing GaSb content.

Shape variation. The capability of X-STM in determining the QD shape is limited since the $\{110\}$ cleavage plane hits the quantum dot at random lateral positions. ${ }^{26}$ For a truncated pyramid this leads to a systematic overestimation of the vertical aspect ratio. Hence, in our calculations we varied the vertical aspect ratio between 0.05 and 0.42 while keeping the volume constant. The latter is important to eliminate the size quantization effect that would originate from a volume change of the QD. As seen from Fig. 6(b), the hole localization energy exhibits only a moderate variation between about 600 and $660 \mathrm{meV}$ for pure GaSb QDs and between 450 and $550 \mathrm{meV}$ for alloyed QDs, respectively. The increase of hole localization with decreasing height (from 6 to $3 \mathrm{~nm}$ height) for pure GaSb QDs is somewhat counterintuitive and can be traced back to a redistribution of strain from being predominantly hydrostatic for tall QDs to biaxial for flat QDs. For QDs flatter than $3 \mathrm{~nm}$ the large $z$-quantization dominates, leading to the expected decrease of localization energy. The $z$-quantization effect is significantly more pronounced for the alloyed QDs, since the overall strain is much smaller, as the lattice mismatch is only half that of pure GaSb QDs.

Influence of the chosen material parameters. The most critical parameter for the localization energy is the valenceband offset between GaAs and GaSb. The classical literature value of $830 \mathrm{meV}^{34}$ was questioned already in the work of North et al., ${ }^{20}$ who found that the localization energies are systematically overestimated. We encountered the same issue and resorted to the value proposed by Wei et al. ${ }^{35}$ who calculated a band offset of $580 \mathrm{meV}$. For all other parameters we followed the recommendations outlined in the paper of Vurgaftman et al. ${ }^{33}$

When comparing the theoretical results with the experimental data, we find that the parameter space used in the calculation leads to localization energies which are in good agreement with the value determined by DLTS. If we assume that the average QD in the ensemble is of high purity ( $\sim 100 \% \mathrm{GaSb}$ content $)$ and has a truncated-pyramid shape, then further constrain the height to $\sim 4-6 \mathrm{~nm}$, the resulting localization energies for such QDs lie within a margin of $\pm 35 \mathrm{meV}$ around a value of $645 \mathrm{meV}$, which is slightly higher than the experimental value of $609 \mathrm{meV}$, but still in good agreement. The calculated value in the work of Marent et al. ${ }^{6}$ for similar QDs yielded a localization energy of $853 \mathrm{meV}$, which is much higher than the values which are calculated in this work. The reason for this large deviation is the use of the classical literature value for the valence-band offset in the calculation. From our experimental data we can clearly see that this value is too high, and the value of Wei et al. ${ }^{35}$ should be used.

\section{CONCLUSION}

We have studied the structural and electronic properties of GaSb QDs in GaAs by X-STM and DLTS. The GaSb QDs are observed to be of high purity ( $\sim 100 \% \mathrm{GaSb}$ content $)$ and 
their shape is that of a truncated pyramid. The average size of the QDs can be constrained to $\sim 4-6 \mathrm{~nm}$ in height and 9-14 $\mathrm{nm}$ in base length. The localization energy of the QD ensemble determined by charge-selective DLTS was $609 \mathrm{meV}$ with an apparent capture cross section of $\sigma_{\infty}=1 \times 10^{-12} \mathrm{~cm}^{2}$. Based on the structural data the QDs were modeled by 8-band $\mathbf{k} \cdot \mathbf{p}$ theory which yielded localization energies of $\sim 490$ $685 \mathrm{meV}$ for heights between 1.4 and $6.3 \mathrm{~nm}$ for $100 \% \mathrm{GaSb}$ content and a constant vertical aspect ratio of 0.2 , and about $100 \mathrm{meV}$ less for just $50 \% \mathrm{GaSb}$ content. When varying the aspect ratio while keeping the volume constant only moderate changes in localization energy are observed. The measured hole localization energy (609 meV) was found to be in good agreement with the calculated values. Our results show that for $\mathrm{GaSb} / \mathrm{GaAs}$ the valence-band offset of $580 \mathrm{meV}$ proposed by Wei et al. ${ }^{35}$ should be used in calculations.

\section{ACKNOWLEDGMENTS}

The authors gratefully acknowledge financial support by the European Commission in the framework of NanoSciE+ Project QD2D (DFG Contract No. BI284/30-1, EPSRC Contract No. EP/H006419, and FOM Contract No. 09NSE0). Additional support comes from DFG Contract No. BI284/291 , as well as the Royal Society-Brain Mercer Feasibility Award (UK), and BMBF via the VIP program Project HOFUS. *nowozin@sol.physik.tu-berlin.de

${ }^{\dagger}$ Also at King Abdulaziz University, Jeddah 21589, Saudi Arabia.

${ }^{1}$ D. Bimberg, M. Grundmann, and N. N. Ledentsov, Quantum Dot Heterostructures (John Wiley \& Sons, Chichester, 1998).

${ }^{2}$ C.-K. Sun, G. Wang, J. E. Bowers, B. Brar, H.-R. Blank, H. Kroemer, and M. H. Pilkuhn, Appl. Phys. Lett. 68, 1543 (1996).

${ }^{3}$ H. Born, L. Müller-Kirsch, R. Heitz, A. Hoffmann, and D. Bimberg, Phys. Status Solidi B 228, R4 (2001).

${ }^{4}$ K. Gradkowski, N. Pavarelli, T. J. Ochalski, D. P. Williams, J. Tatebayashi, G. Huyet, E. P. OReilly, and D. L. Huffaker, Appl. Phys. Lett. 95, 061102 (2009).

${ }^{5}$ R. B. Laghumavarapu, A. Moscho, A. Khoshakhlagh, M. El-Emawy, L. F. Lester, and D. L. Huffaker, Appl. Phys. Lett. 90, 173125 (2007).

${ }^{6}$ A. Marent, M. Geller, A. Schliwa, D. Feise, K. Pötschke, D. Bimberg, N. Akçay, and N. Öncan, Appl. Phys. Lett. 91, 242109 (2007).

${ }^{7}$ M. Geller, A. Marent, T. Nowozin, D. Bimberg, N. Akçay, and N. Öncan, Appl. Phys. Lett. 92, 092108 (2008).

${ }^{8}$ A. Marent, T. Nowozin, M. Geller, and D. Bimberg, Semicond. Sci. Technol. 26, 014026 (2011).

${ }^{9}$ F. Hatami, N. N. Ledentsov, M. Grundmann, J. Böhrer, F. Heinrichsdorff, M. Beer, D. Bimberg, S. S. Ruvimov, P. Werner, U. Gösele, J. Heydenreich, U. Richter, S. V. Ivanov, B. Y. Meltser, P. S. Kop'ev, and Z. I. Alferov, Appl. Phys. Lett. 67, 656 (1995).

${ }^{10}$ B. M. Kinder and E. M. Goldys, Appl. Phys. Lett. 73, 1233 (1998).

${ }^{11}$ L. Müller-Kirsch, R. Heitz, U. W. Pohl, D. Bimberg, I. Häusler,

H. Kirmse, and W. Neumann, Appl. Phys. Lett. 79, 1027 (2001).

${ }^{12}$ H. Sunamura, Y. Shiraki, and S. Fukatsu, Appl. Phys. Lett. 66, 953 (1996).

${ }^{13}$ R. A. Hogg, K. Suzuki, K. Tachibana, L. Finger, K. Hirakawa, and Y. Arakawa, Appl. Phys. Lett. 72, 2856 (1998).

${ }^{14}$ F. Hatami, M. Grundmann, N. N. Ledentsov, F. Heinrichsdorff, R. Heitz, J. Böhrer, D. Bimberg, S. S. Ruvimov, P. Werner, V. M. Ustinov, P. S. Kop'ev, and Z. I. Alferov, Phys. Rev. B 57, 4635 (1998).

${ }^{15}$ M. Hayne, O. Razinkova, S. Bersier, R. Heitz, L. Müller-Kirsch, M. Geller, D. Bimberg, and V. V. Moshchalkov, Phys. Rev. B 70, 081302 (2004).

${ }^{16}$ R. Timm, H. Eisele, A. Lenz, S. K. Becker, J. Grabowski, T. Y. Kim, L. Müller-Kirsch, K. Pötschke, U. W. Pohl, D. Bimberg, and M. Dähne, Appl. Phys. Lett. 85, 5890 (2004).
${ }^{17}$ R. Magno, B. R. Bennett, and E. R. Glaser, J. Appl. Phys. 88, 5843 (2000).

${ }^{18}$ M. Geller, C. Kapteyn, L. Müller-Kirsch, R. Heitz, and D. Bimberg, Appl. Phys. Lett. 82, 2706 (2003).

${ }^{19}$ M. E. Rubin, H. R. Blank, M. A. Chin, H. Kroemer, and V. Narayanamurti, Appl. Phys. Lett. 70, 1590 (1997).

${ }^{20}$ S. M. North, P. R. Briddon, M. A. Cusack, and M. Jaros, Phys. Rev. B 58, 12601 (1998).

${ }^{21}$ M. Hayne, J. Maes, S. Bersier, V. V. Moshchalkov, A. Schliwa, L. Müller-Kirsch, C. Kapteyn, R. Heitz, and D. Bimberg, Appl. Phys. Lett. 82, 4355 (2003).

${ }^{22}$ D. Bimberg, F. Guffarth, K. Pötschke, S. Rodt, and A. Schliwa, Mater. Sci. Eng., C 25, 698 (2005).

${ }^{23}$ O. Stier, M. Grundmann, and D. Bimberg, Phys. Rev. B 59, 5688 (1999).

${ }^{24}$ A. Schliwa, M. Winkelnkemper, and D. Bimberg, Phys. Rev. B 76, 205324 (2007).

${ }^{25}$ E. P. Smakman, J. K. Garleff, R. J. Young, M. Hayne, P. Rambabu, and P. Koenraad, Appl. Phys. Lett. 100, 142116 (2012).

${ }^{26}$ O. Flebbe, H. Eisele, T. Kalka, F. Heinrichsdorff, A. Krost, D. Bimberg, and M. Dähne-Prietsch, J. Vac. Sci. Technol. B 17, 1639 (1999).

${ }^{27}$ D. V. Lang, J. Appl. Phys. 45, 3023 (1974).

${ }^{28} \mathrm{P}$. Blood and J. W. Orton, The Electrical Characterization of Semiconductors: Majority Carriers and Electron States (Academic Press, London, 1992).

${ }^{29}$ C. M. A. Kapteyn, M. Lion, R. Heitz, D. Bimberg, P. N. Brunkov, B. V. Volovik, S. G. Konnikov, A. R. Kovsh, and V. M. Ustinov, Appl. Phys. Lett. 76, 1573 (2000).

${ }^{30}$ P. Omling, L. Samuelson, and H. G. Grimmeis, J. Appl. Phys. 54, 5117 (1983).

${ }^{31}$ D. S. Day, M. Y. Tsai, B. G. Streetman, and D. V. Lang, J. Appl. Phys. 50, 5093 (1979)

${ }^{32}$ C. M. A. Kapteyn, M. Lion, R. Heitz, D. Bimberg, C. Miesner, T. Asperger, and G. Abstreiter, Appl. Phys. Lett. 77, 4169 (2000).

${ }^{33}$ I. Vurgaftman, J. R. Meyer, and L. R. Ram-Mohan, J. Appl. Phys. 89, 5815 (2001).

${ }^{34}$ C. G. Van de Walle, Phys. Rev. B 39, 1871 (1989).

${ }^{35}$ S.-H. Wei and A. Zunger, Appl. Phys. Lett. 72, 2011 (1998). 